\title{
La evaluación de los aprendizajes de los estudiantes en el marco del Espacio Europeo de Educación Superior (EEES): sus prácticas, preferencias y evolución
}

\author{
Javier Argos, Pilar Ezquerra, José Manuel Osoro, Laurentino \\ Salvador y Ana Castro \\ Universidad de Cantabria (España)
}

\begin{abstract}
El Espacio Europeo de Educación Superior (EEES) comporta, al menos a nivel teórico, cambios sustanciales en los planteamientos formativos desarrollados que se concretan en diferentes ámbitos. Uno de éstos, el de la evaluación de los aprendizajes de los estudiantes, se convierte en el foco del trabajo que presentamos. Los planteamientos y resultados del mismo se enmarcan en un proyecto I+D+i (EDU 2009-13195) titulado "Enfoques de aprendizaje de los estudiantes universitarios, metodologías docentes y contextos institucionales al inicio, intermedio y final de la carrera en el marco de implantación de los nuevos títulos". Concretamente, nos centramos en la evaluación de los aprendizajes analizando, tanto los formatos o modalidades utilizadas, como las preferencias que los estudiantes manifiestan sobre ellas, en diferentes cursos y titulaciones. Asimismo, pretendemos aportar algo de luz acerca de cómo dichas preferencias varían dependiendo del enfoque de aprendizaje de cada estudiante (profundo o superficial) y, también, de la evolución que se produce en los alumnos a lo largo de los diferentes cursos de sus estudios de grado.
\end{abstract}

Palabras clave: Educación Superior, enfoques de aprendizaje, metodologías docentes, evaluación educativa, Espacio Europeo de Educación Superior.

Assessment of students' learning outcomes in the EHEA: their practices, preferences and evolution. The European Higher Education Area implies, at least theoretically, substantial changes in the developed educational approaches specified in several areas. Among these the assessment of students' learning is the focus of this article. The approaches and results that are exposed here are framed in an Educational Research Project entitled "Learning approaches of the university students, teaching strategies and institutional contexts to the beginning, half and end of career in the process of implantation of the new degrees". Specifically, we focus on the learning assessment, analyzing both the formats or modalities of this kind of evaluation as well as the preferences which the students have concerning them in the context of different courses and university degrees. Furthermore, we try to somewhat clarify how these preferences could vary depending on the learning approach of each student (deep or superficial) and, also, on the students' evolution along the different courses of their degree.

Key words: Higher Education, learning approaches, teaching methodologies, educational assessment, European Higher Education Area.

Correspondencia: Javier Argos. Departamento de Educación. Universidad de Cantabria. Avda. de los Castros, s/n. C.P. 39005. Santander (España). E-mail: argosj@unican.es 
El proceso de investigación que estamos desarrollando nos permite estudiar, por un lado, los enfoques de aprendizaje que los estudiantes universitarios utilizan en la carrera y, por otro, relacionar éstos con los planteamientos metodológicos y con las estrategias didácticas que los docentes universitarios despliegan en los procesos de enseñanza-aprendizaje. El Espacio Europeo de Educación Superior (EEES) nos propone cambios significativos en relación con todos estos aspectos.

En concreto, en el presente trabajo abordamos la relación que tienen los diferentes enfoques de aprendizaje de los estudiantes con los procedimientos de evaluación preferidos por éstos y los utilizados por el profesorado.

\section{Marco teórico}

Son abundantes las referencias efectuadas en la literatura nacional e internacional en torno a los estudios sobre los enfoques de aprendizaje. En el ámbito internacional, buena parte de ellos, surgen de los trabajos de Biggs (Biggs, 1988, 1993 y 2005, Biggs et al., 2001), aunque en el ámbito nacional han sido también amplias las contribuciones a este tema (Barca, 1999; Cabanach, 1997; De la Fuente y Martínez, 2003; Gargallo et al., 2009, 2011 y 2012; Hernández, 1999).

Los enfoques de aprendizaje integran tanto la motivación que el estudiante tiene para realizar una tarea como las estrategias que va a utilizar para conseguirlo. Se trata de algo complejo, dinámico y continuo que afecta a procesos profundos del conocimiento (metacognición) y a la utilización, en la práctica, de las estrategias necesarias para la consecución de los objetivos de aprendizaje.

En el marco del proyecto de investigación en el que estamos implicados, en trabajos anteriores (Argos et al., 2011a y 2011b; Castro et al., 2012; Goikoetxea et al., 2010; Salvador et al., 2011; San Fabián et al., 2010) hemos abordado la conceptualización sobre los enfoques de aprendizaje y su vinculación con las estrategias y estilos de enseñanza que los docentes utilizan en sus aulas. En los trabajos citados hemos dimensionado y definido la profundidad del enfoque como un continuo bipolar que se puede desplazar, incluso en el mismo sujeto, desde lo más superficial a lo más profundo (desde la motivación extrínseca, el interés escaso por la materia, la memorización excesiva sin acompañamiento comprensivo, cierto miedo al fracaso hasta la motivación intrínseca, el interés por las materias, la utilización de estrategias de comprensión, etc.).

Debemos de señalar, no obstante, que no existe una vinculación directa entre los enfoques de aprendizaje, las estrategias que el estudiante utiliza para enfrentarse a las tareas y los resultados obtenidos. Podría pensarse que en el ámbito universitario el estudiante con mayor profundidad en su enfoque utilizaría siempre estrategias más comprensivas y obtendría mejores resultados pero, como ya hemos indicado en un trabajo previo, esto no es así necesariamente (Castro et al., 2012). Creemos que el 
estudiante no se enfrenta en todo momento a las tareas con un enfoque profundo, sino que algunas tareas las podrá afrontar de manera profunda y otras de manera superficial. Esto nos hace pensar que la intensidad del enfoque aplicada tiene que ver con el tipo de tareas que debe realizar y, por lo tanto, también con los métodos y procedimientos didácticos y los requisitos de evaluación que el profesorado plantea en sus clases.

Teniendo en cuenta lo anterior, podemos decir que los enfoques no son estables e inalterables, sino que dependen del contexto y de la tarea a realizar. En este sentido, algunos autores (Cabanach, 1997; Gargallo et al., 2012 y Ezquerra et al., 2012) indican que las metodologías de enseñanza utilizadas por el profesorado condicionan los enfoques utilizados por los estudiantes. Dicho de otra forma, aquellas metodologías más cercanas al aprendizaje, con diversidad de métodos y procedimientos de evaluación requerirían una mayor profundidad del enfoque del aprendizaje mientras que aquellas otras más centradas en la enseñanza, es decir, con métodos más expositivos y procedimientos únicos de evaluación, se relacionarían más con posicionamientos superficiales por parte de los estudiantes.

En el siguiente esquema presentamos los aspectos esenciales del modelo en que enmarcamos el trabajo.

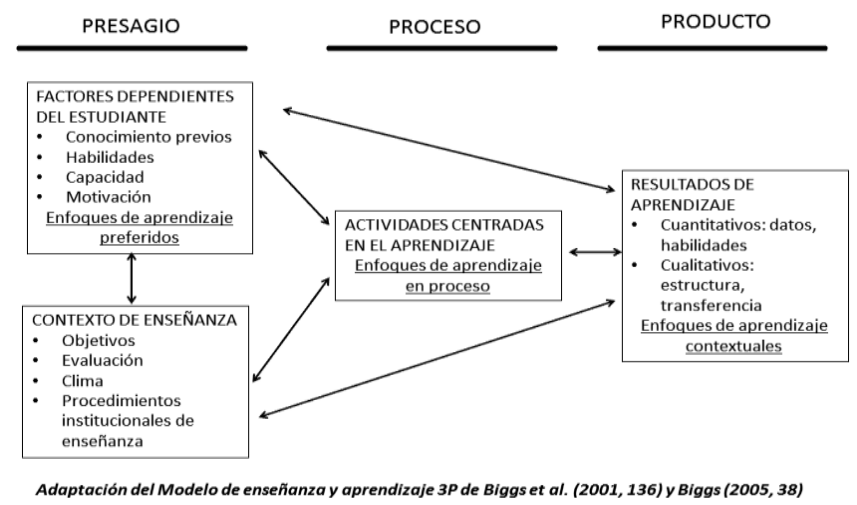

El proceso de implementación del Espacio Europeo de Educación Superior (EEES) ha sido una magnífica oportunidad para que la institución formativa y su profesorado replantearan sus estilos docentes. Como hemos señalado en otros trabajos previos (Argos et al., 2007 y 2011a; Osoro et al., 2011 y Salvador et al., 2011) el marco de actuación del EEES comporta importantes retos tanto para las universidades como para sus docentes, constatando, desde una perspectiva general, las vinculaciones existentes entre los enfoques de aprendizaje del alumnado y las preferencias y usos de las metodologías, prácticas y procedimientos de evaluación por parte del profesorado. 
Con la pretensión de profundizar en el referido ámbito de la evaluación, en el presente trabajo presentamos la relación entre los procedimientos de evaluación preferidos por los estudiantes y los utilizados por los docentes con la profundidad del enfoque que utilizan aquellos en sus estudios.

Si focalizamos ahora nuestra mirada en la evaluación es debido a que, desde nuestro punto de vista, constituye uno de los elementos fundamentales en relación con los cambios que se nos proponen desde el EEES. Una de las consecuencias del replanteamiento metodológico que plantea el EEES es la reconsideración y diversificación de los procedimientos para evaluar el aprendizaje del alumnado. En general, podemos decir con De Miguel (2005) que las modalidades organizativas más magistrales se asocian más con estrategias evaluativas clásicas (pruebas objetivas, tipo test, de desarrollo), mientras que la evaluación a través de trabajos o proyectos se relaciona más con métodos de aprendizaje basados en problemas, métodos cooperativos, etc.

No se trata, sin embargo, de un problema de multiplicidad de métodos didácticos ni de cantidad de procedimientos evaluativos, sino que el cambio sugerido se dirige a la propia filosofía de la evaluación, es decir, el paso hacia un modelo de evaluación formativa en detrimento de la sumativa, una apuesta por la evaluación continua en relación a la evaluación final y la posibilidad de utilizar procedimientos que incorporen al alumnado como agente de evaluación (auto y heteroevaluación). Es interesante en este sentido la aportación de Fraile y Cornejo (2012: 2) que sitúa la evaluación en un plano formativo como alternativa al uso tradicional de la evaluación sumativa más centrada en la búsqueda de la eficacia y la calificación final. Compartimos con estos autores la idea de que la evaluación formativa tiene un calado más profundo que la mera utilización de procedimientos evaluativos que abunden en esta perspectiva. Para ellos, el carácter formativo de la evaluación contribuirá a la "participación activa de los estudiantes siendo el docente un facilitador de este proceso, impulsando valores educativos como son, entre otros la honradez, la dignidad y la autocrítica".

\section{MÉTODO}

\section{Participantes}

La muestra objeto de estudio la conforman diferentes titulaciones de las universidades de Cantabria, Oviedo y País Vasco. En la Universidad de Cantabria se comenzó el estudio con las titulaciones de Historia, Geografía y Medicina, incorporándose en el curso 2010/11 los grados de Maestro de Educación Infantil y de Educación Primaria, año en el que se iniciaba su proceso de implementación.

Los datos de los estudiantes de la Universidad de Cantabria que recogemos y analizamos en este trabajo pertenecen a alumnos de primero, segundo y tercer curso (ver 
tablas 1, 2 y 3), no estando aún procesados los del presente curso académico. Cuando esto ocurra podremos contar, al menos en algunas de las titulaciones de la muestra, con datos de todos los cursos de los grados.

Tabla 1. Distribución de los estudiantes de la Universidad de Cantabria según género y curso

\begin{tabular}{rrrrrrrr}
\hline & \multicolumn{3}{c}{ Género } & \multicolumn{4}{c}{ Curso } \\
\cline { 2 - 8 } & Varón & Mujer & Total & $1^{\circ}$ & $2^{\circ}$ & $3^{\circ}$ & Total \\
\hline $\mathrm{N}$ & 324 & 803 & 1127 & 770 & 328 & 33 & 1131 \\
\hline$\%$ & 28.7 & 71.3 & 100 & 68.1 & 29 & 2.9 & 100 \\
\hline
\end{tabular}

Tabla 2. Distribución de los estudiantes de la Universidad de Cantabria según Facultad

\begin{tabular}{lcc}
\hline \multicolumn{1}{c}{ Facultad } & $N$ & $\%$ \\
\hline Educación & 727 & 64.3 \\
\hline Filosofía y Letras & 333 & 29.4 \\
\hline Medicina & 71 & 6.3 \\
\hline Total & 1131 & 100 \\
\hline
\end{tabular}

Tabla 3. Distribución de los estudiantes de la Universidad de Cantabria según Titulación

\begin{tabular}{lrc}
\hline \multicolumn{1}{c}{ Titulación } & $N$ & $\%$ \\
\hline Geografía & 97 & 8.6 \\
\hline Historia & 236 & 20.9 \\
\hline Maestro Educación Infantil & 304 & 26.9 \\
\hline Maestro Educación Primaria & 423 & 37.4 \\
\hline Medicina & 71 & 6.3 \\
\hline Total & 1131 & 100 \\
\hline
\end{tabular}

\section{Diseño y procedimiento}

El estudio que estamos desarrollando utiliza una metodología de tipo mixto, integrando procedimientos y técnicas tanto cuantitativas (cuestionarios a estudiantes y a docentes) como cualitativas (grupos de discusión con estudiantes y con docentes, por separado) y ostenta un carácter longitudinal. Así, comienza en el curso 2009/10 con los estudiantes que inician sus estudios y continúa en el presente 2012/13.

Dicho planteamiento metodológico permite llevar a cabo sendos procesos de triangulación, tanto de fuentes de información (estudiantes, profesorado y equipos decanales) como de técnicas (cuestionarios, grupos de discusión y /o entrevistas) que nos permitan el análisis y la posterior interpretación de las dimensiones y variables objeto de estudio.

\section{Dimensiones y variables}

Para conocer la perspectiva de los estudiantes se diseñó un cuestionario que, como ya hemos comentado en anteriores trabajos (Argos et al., 2011a y 2011b), estuvo compuesto inicialmente por 198 ítems, que atendían a las diferentes variables y dimensiones establecidas. Posteriormente, tras la primera fase de recogida de información, el instrumento para los estudiantes de primer curso se redujo hasta los 177 ítems con el objeto de facilitar su cumplimentación así como para desechar algunas 
preguntas abiertas a las que contestaban muy pocos. Asimismo, en el cuestionario para el alumnado de los cursos posteriores se prescindió de los ítems iniciales de identificación de cada estudiante quedando conformado por 168 ítems.

También se llevaron a cabo grupos de discusión (cuyo análisis, por limitaciones de espacio, no presentamos aquí) con estudiantes de las titulaciones de Maestro en Educación Infantil, Maestro en Educación Primaria, Historia y Geografía de la Universidad de Cantabria. Se contemplaron los ámbitos temáticos y las dimensiones que estaban recogidos en los cuestionarios para facilitar el proceso de triangulación de la información.

Las variables primarias (los ítems del cuestionario) se sometieron a un proceso estadístico reductor para agruparlas en dimensiones o en áreas más parsimoniosas. La fundamentación pormenorizada del establecimiento de estas dimensiones la hemos descrito en otros trabajos (Ezquerra et al., 2012 y Castro et al., 2012). Teniendo en cuenta el modelo que nos sirve de marco, relacionamos, de forma sucinta, algunas de las dimensiones procesuales más relevantes para, seguidamente, contemplar con mayor detalle las referidas a los enfoques de aprendizaje de los estudiantes y a las preferencias y usos en relación con la evaluación.

Estrategias metodológicas: de orientación práctica (estudio de casos, prácticas de laboratorio, resolución de problemas y simulaciones), técnica expositiva (combinada con el uso de organizadores previos, esquemas... y con ejercicios individuales durante las clases), ejercicios en grupo (utilización de dinámicas grupales con realización de ejercicios en grupo durante las clases) y seminario.

Recursos: de lápiz y papel (manual de la asignatura y/o apuntes entregados por el profesor, encerado y apuntes tomados por los estudiantes) y digitales (presentaciones en PowerPoint, programas de ordenador, recursos de internet y vídeos).

Evaluación: Es el bloque en el que se centrará el presente estudio y que se configura en las cuatro dimensiones básicas que presentamos a continuación, estando las tres primeras más vinculadas a técnicas de evaluación específicas mientras que, la cuarta, se centra en la modalidad evaluativa.

- Trabajos y casos: la evaluación se centra fundamentalmente en trabajos prácticos, memorias o informes, proyectos de investigación, pruebas de ejecución de tareas reales o simuladas y ejercicios o casos prácticos. Consta de 8 ítems.

- Pruebas abiertas y proyectos: la evaluación se materializa en preguntas abiertas o de desarrollo y en trabajos o proyectos en grupo o individuales. Conformado por 6 ítems.

- Pruebas cerradas: la evaluación se basa en pruebas con preguntas cortas y en pruebas objetivas. Integrado por 4 ítems.

- Evaluación continua. Compuesto por 2 ítems. 
Estrategias y enfoques de aprendizaje: integramos las estrategias de aprendizaje presentadas por Navaridas (2004) y redefinidas en función de los análisis factoriales realizados con los enfoques de aprendizaje establecidos por Biggs y adaptados en el ámbito español por sendos estudios de Hernández Pina (1999 y 2003) y De la Fuente y Martínez (2003). Así hemos establecido las siguientes dimensiones:

- Estrategia proactiva de organización y apoyo (el estudiante subraya, resume, esquematiza y clasifica el contenido a aprender y revisa y amplia las notas tomadas en clase).

- Estrategia de elaboración (necesidad de tener una visión global de su contenido antes de estudiarlo, asociando lo aprendido con lo ya conocido o experimentado, trata de buscar aplicaciones a los nuevos conocimientos adquiridos).

- Estrategia de autocontrol o adaptativa (antes de preparar una asignatura el estudiante trata de saber cómo son los exámenes, adaptando su forma de estudio al estilo y a las exigencias de cada docente y modificando su forma de estudio a los resultados obtenidos).

- Y estrategia de repetición (el estudiante suele recitar el contenido de la materia repitiéndolo tantas veces como sea necesario hasta memorizarlo para la evaluación. Durante las clases intenta copiar las exposiciones del profesor de forma literal).

- Enfoque de Aprendizaje elaborado a partir del RSPQ-2F (adaptado por De la Fuente y Martínez, 2003) que comprende dos escalas:

- Enfoque Profundo (Deep Approach: DA) integrada por las subescalas Motivación profunda y Estrategia profunda.

- Enfoque Superficial (Superficial Approach: SA) que consta de las subescalas Motivación superficial y Estrategia superficial.

- Intensidad de la profundidad del enfoque: es el resultado de cuantificar la puntuación de cada estudiante en un continuo bipolar que va desde lo superficial a lo profundo o, lo que es lo mismo, desde lo muy poco profundo a lo muy profundo. Hemos operado calculando la puntuación diferencial existente entre el enfoque profundo (DA) y el superficial (SA) y, para evitar las puntuaciones negativas, hemos desplazado el 0 al punto mínimo de la muestra. Así, cuanto más elevada es la puntuación obtenida mayor será la profundidad del enfoque de aprendizaje adoptado por el estudiante (motivación extrínseca vs. intrínseca, interés escaso vs. elevado por la materia, memorización excesiva sin acompañamiento comprensivo vs. utilización de estrategias de comprensión y memorización selectiva, presencia vs. ausencia de miedo al fracaso, etc.).

- Enfoque profundo-estratégico: para obtener esta puntuación hemos utilizado la técnica del análisis discriminante tomando como variable de agrupamiento la profundidad del enfoque categorizada e incluyendo las variables primarias del RSPQ-2F (ítems 32 a 51) y las indicadas por Navaridas (2004) que se corresponden con los ítems 
106 a 121 de nuestro cuestionario. El procedimiento estadístico asigna una puntuación de 1 a 5 a cada estudiante (muy baja/ baja/ media/ alta/ muy alta).

\section{RESULTADOS}

Diferencias genéricas entre sobre preferencias y usos en evaluación

Utilizando la prueba de T-Test con datos pareados, hemos sometido a contraste las preferencias que muestran y los usos que perciben cada uno de los estudiantes en relación con la evaluación de sus aprendizajes. Todas las diferencias de medias obtenidas entre ambos aspectos resultan estadísticamente significativas $(p \leq .05)$ salvo en los elementos referidos a las pruebas objetivas y a las preguntas abiertas a desarrollar.

Gráfico 1. Representación de las medias en preferencias y usos evaluativos

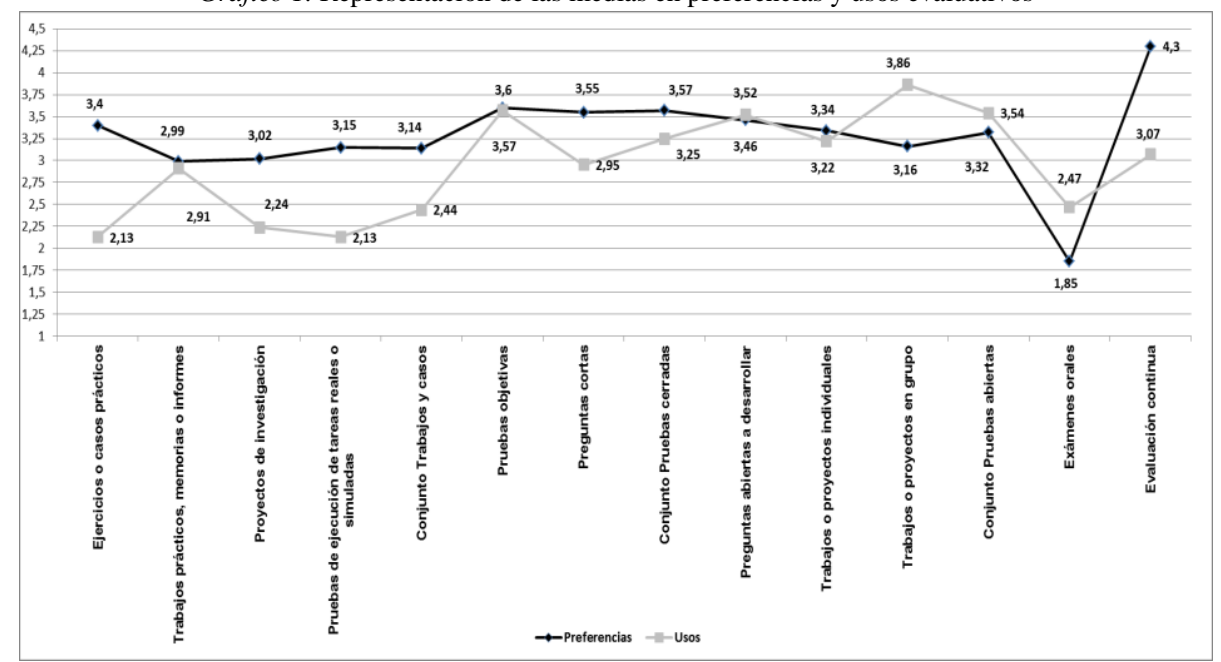

Así podemos decir que, en general, el alumnado prefiere una evaluación continua que incorpore técnicas o procedimientos de distinto tipo. La media de sus preferencias está por encima de la media de usos en lo referido a ejercicios o casos prácticos (1.27), proyectos de investigación (0.79), pruebas de ejecución de tareas reales o simuladas (1.01), conjunto trabajos y casos (0.70), preguntas cortas (0.61), conjunto pruebas cerradas $(0.33)$ y evaluación continua (1.23).

Hay una coincidencia importante en preferencias y usos en los siguientes procedimientos: trabajos prácticos $(0.08)$, memorias o informes $(0.08)$, pruebas objetivas (0.02), trabajos o proyectos individuales $(0.12)$. 
Por último, predominan los usos sobre las preferencias en el resto de los aspectos, esto es, en los trabajos o proyectos en grupo (0.70), en el conjunto de pruebas abiertas (0.47) y en los exámenes orales (0.62). Dicho de otro modo, en estos casos el profesorado utiliza estas estrategias evaluativas con mayor frecuencia que la preferida por el alumnado.

Diferencias entre preferencias y usos en evaluación según los distintos agrupamientos de los estudiantes

En el presente apartado pretendemos mostrar la existencia, en su caso, de diferencias estadísticamente significativas entre los distintos agrupamientos efectuados atendiendo a diferentes características de los estudiantes (sexo, curso, titulación, enfoque de aprendizaje y enfoque profundo-estratégico de aprendizaje) y a las variables secundarias que hemos descrito con anterioridad y relativas tanto a preferencias como a usos evaluativos: trabajos y casos (trabajos prácticos, memorias o informes, proyectos de investigación, pruebas de ejecución de tareas reales o simuladas y, ejercicios o casos prácticos), pruebas abiertas y proyectos (preguntas abiertas, de desarrollo, trabajos o proyectos en grupo e individuales), pruebas cerradas (preguntas cortas y pruebas objetivas) y evaluación continua.

Los comentarios que efectuamos a continuación se limitarán, fundamentalmente, a aquellos casos en los que los resultados resultaron estadísticamente significativos $(p \leq .05)$ mediante contraste de medias ONEWAY y post hoc de Scheffé.

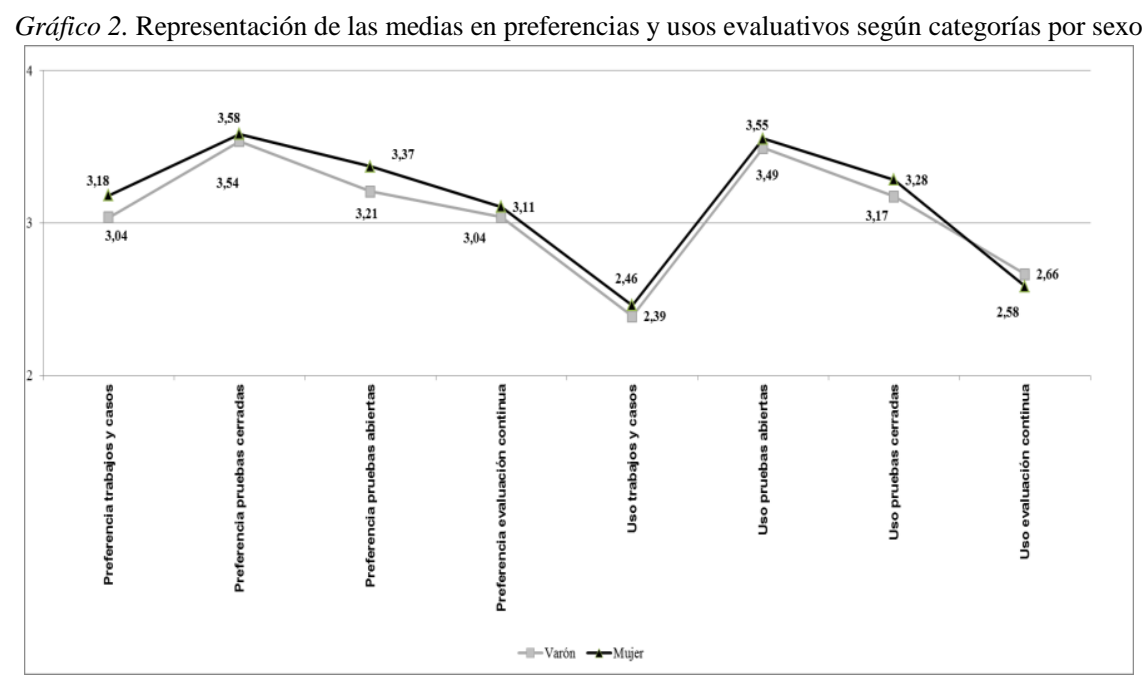

Atendiendo a la variable Sexo, se constata lo siguiente: 
- Preferencias: en general, las mujeres, prefieren ser evaluadas, a diferencia de los varones, mediante trabajos y casos $(0.16)$ y mediante pruebas abiertas $(0.16)$. No hay diferencias estadísticamente significativas entre ambos en el caso de las pruebas cerradas y la evaluación continua.

- Usos: al tratarse de una cuestión de hecho y no de opinión, como era de esperar, las diferencias no resultaron estadísticamente significativas.

- Intensidad de la profundidad del enfoque y enfoque profundo-estratégico: en ambos casos las diferencias resultaron significativas, constatándose en las alumnas una mayor intensidad en la profundidad de enfoque que en los alumnos varones de la muestra.

Por lo que respecta al Curso de los estudiantes, es de destacar:

- Sólo hemos encontrado diferencias estadísticamente significativas en el caso de la preferencia por la evaluación continua en los alumnos de primero frente a los de segundo y tercero. A medida que avanzan los cursos desciende significativamente la preferencia por la evaluación continua $(3.15,2.96$ y 2.79 respectivamente). No se han encontrado diferencias estadísticamente significativas en el resto de las variables evaluativas.

- También hay diferencias significativas en lo que respecta a la intensidad de la profundidad del enfoque, de manera que ésta va descendiendo progresivamente desde primer curso (36.41) hasta tercero (24.82).

Gráfico 3. Representación de las medias en preferencias y usos evaluativos según curso

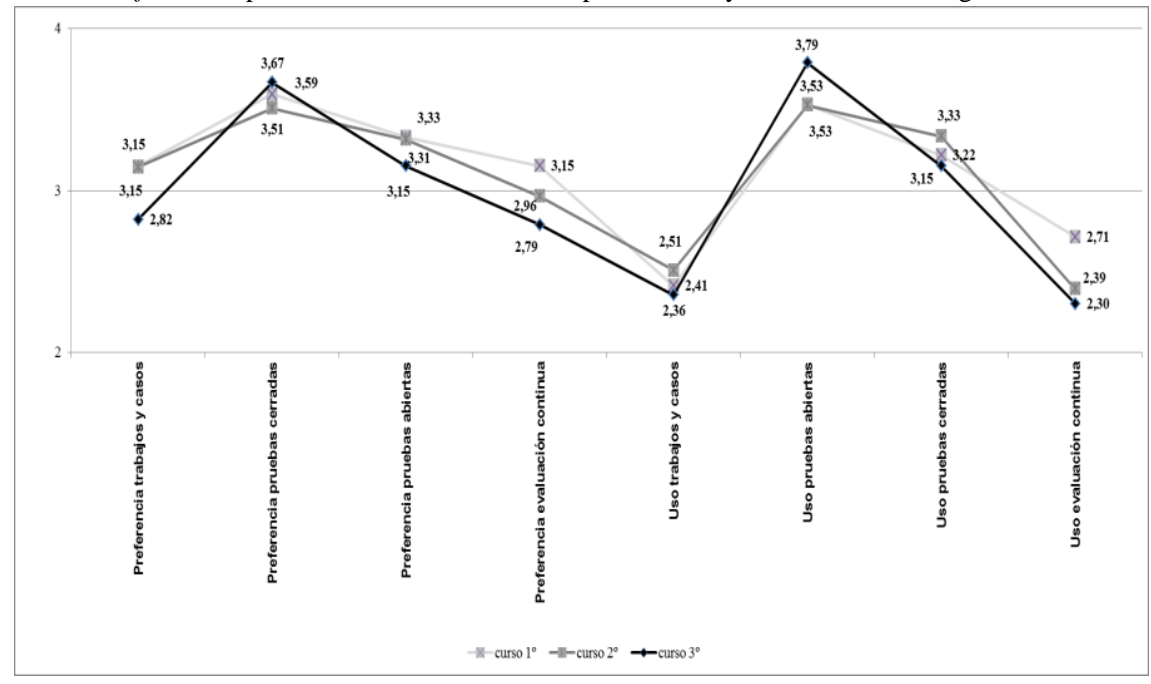

En cuanto a la variable Titulación, podemos apuntar los siguientes datos: 
- Preferencias: en todas las variables secundarias las diferencias resultaron estadísticamente significativas. En concreto, éstas se aprecian entre la titulación de Maestro de Ed. Infantil y las de Historia y Geografía en la preferencia de los estudiantes de la primera por la evaluación mediante trabajos y casos. Asimismo, en la mayor preferencia por las pruebas cerradas observada en los estudiantes de Medicina frente a los de las restantes titulaciones. Por su parte, la preferencia por las pruebas abiertas es mayor en las titulaciones de Maestro de Ed. Primaria, de Maestro de Ed. Infantil y de Historia que en las de Medicina y Geografía. Por último, se constatan diferencias significativas en la evaluación continua, apareciendo una mayor preferencia en Maestro Ed. Primaria, Maestro Ed. Infantil e Historia que en Geografía.

- Usos: en este caso todas las diferencias de medias resultaron estadísticamente significativas, pudiendo establecerse las siguientes diferencias por titulaciones:

- El uso de trabajos y casos como procedimientos de evaluación es más frecuente en Geografía y en Maestro de Ed. Infantil que en Historia y Medicina. Ocurre lo mismo en la titulación de Maestro de Ed. Primaria respecto a la de Medicina.

- La utilización de pruebas abiertas es más frecuente en Geografía que en Medicina, en Historia más que en el resto de titulaciones y en Maestro de Ed. Primaria más que en Geografía, Maestro de Ed. Infantil y Medicina.

- El uso de pruebas cerradas es más común en Medicina que en las restantes titulaciones. Por su parte, en Maestro de Ed. Primaria y Maestro de Ed. Infantil dicho uso es más frecuente que en Historia.

- El predominio del uso de la evaluación continua se produce en Geografía frente Medicina, Historia y Maestro de Ed. Infantil. Igualmente, en la titulación de Maestro de Ed. Primaria es más frecuente el uso de la evaluación continua que en Medicina.

- Intensidad de la profundidad del enfoque: nos encontramos con diferencias estadísticamente significativas solamente en el caso de Geografía (28.14) que presenta una menor profundidad de enfoque que el resto de las titulaciones (35.85).

- Enfoque profundo y estratégico: las diferencias estadísticamente significativas aparecen en los siguientes casos:

- En la titulación de Geografía aparecen más alumnos de los que cabría esperar con un enfoque muy poco profundo y, lógicamente, menos de los que cabría esperar con un enfoque profundo alto.

- En el grado de Historia hay menos alumnos de los que cabría esperar con enfoque profundo-estratégico medio y más en el enfoque muy alto.

- En Maestro de Ed. Primaria aparece un número mayor de estudiantes del que cabría esperar con enfoque profundo muy bajo y bajo y menos de lo que 
cabría esperar con un enfoque profundo alto. Los estudiantes se concentran mayormente en la zona media-alta.

- En Maestro de Ed. Infantil aparecen un número mayor de alumnos del que cabría esperar con enfoque profundo-estratégico bajo a costa de los que cabría esperar con un enfoque profundo alto. Los estudiantes se concentran mayormente en la zona baja y muy baja.

- En Medicina no aparecen diferencias estadísticamente significativas en este aspecto.

Atendiendo a la relación entre datos evaluativos y nivel de enfoque profundoestratégico podemos establecer los siguientes perfiles de preferencias:

- No se han encontrado diferencias significativas entre los grupos de estudiantes en la preferencia por la evaluación mediante pruebas objetivas y con trabajos en grupo.

- Estudiantes con nivel de enfoque profundo-estratégico alto-muy alto: Prefieren los proyectos de investigación, los trabajos prácticos, las preguntas abiertas a desarrollar, los ejercicios o casos prácticos, los trabajos o proyectos individuales y los de grupos, los exámenes orales y la evaluación continua. No les gustan las preguntas cortas.

- Estudiantes con nivel de enfoque profundo-estratégico medio: Prefieren moderadamente la evaluación mediante preguntas cortas, los trabajos y proyectos en grupo, los trabajos prácticos y memorias. No son partidarios de los proyectos de investigación, de la evaluación continua ni de las preguntas abiertas.

- Estudiantes con nivel de enfoque profundo-estratégico muy bajo-bajo: Prefieren las preguntas cortas. No tienen preferencias por el resto de las pruebas: proyectos de investigación, de ejecución, trabajos prácticos, preguntas abiertas, ejercicios o casos prácticos, exámenes orales, trabajos o proyectos individuales, trabajos o proyectos de grupo y la evaluación continua.

\section{APUNTES CONCLUSIVOS}

Como se ha podido apreciar en el análisis de resultados, los estudiantes muestran preferencias evaluativas diferenciadas en función del género, del curso, de la titulación y del enfoque de aprendizaje concreto del estudiante. Las preferencias de los estudiantes son coincidentes con lo propugnado en el marco del Espacio Europeo de Educación Superior en el que la evaluación continua, de carácter formativo, ostenta un mayor protagonismo y se sustenta en procedimientos evaluadores diversos. Los resultados, aquí someramente analizados, podrían sugerir unos usos de la evaluación que estuvieran más en consonancia con los distintos enfoques de aprendizaje de los estudiantes, así como con las peculiaridades de los diferentes marcos personales e institucionales. 
Agradecimientos: Este trabajo se enmarca en un proyecto $\mathrm{I}+\mathrm{D}+\mathrm{i}$ (EDU 2009-13195) titulado "Enfoques de aprendizaje de los estudiantes universitarios, metodologías docentes y contextos institucionales al inicio, intermedio y final de la carrera en el marco de implantación de los nuevos títulos".

\section{REFERENCIAS}

Argos, J., Gárate, M., Monge, J.J., Osoro, J.M. y Salvador, L. (2007). El Espacio Europeo de Educación Superior (EEES). Análisis y reflexión desde la Facultad de Educación. Santander: TGD-Universidad de Cantabria.

Argos, J., Castro, C., Ezquerra, M.P., Osoro, J.M. y Salvador, L. (2011a). Estudio de los contextos de enseñanza y aprendizaje universitarios percibidos por estudiantes y profesores: avance de resultados de la percepción de los estudiantes. En J.J. Maquillon et al. (Coords.), Innovación educativa en la enseñanza formal (pp. 795-804). Murcia: Editum. http://edit.um.es/library/docs/books/9788469428429.pdf (consultado 15/02/2013).

Argos, J., Castro, C., Ezquerra, M.P., Osoro, J.M. y Salvador, L. (2011b). Percepción de los estudiantes universitarios sobre los contextos de enseñanza y aprendizaje en las nuevas titulaciones de Grado. En J. Román, M.A. Carbonero y J. Donoso (Comps.), Educación $y$ desarrollo en una sociedad multicultural (pp. 2665-2681). Madrid: Asociación de Psicología y Educación.

Barca, A. (1999). CEPEA. Cuestionario de Evaluación de Procesos de estudio y Aprendizaje para el alumnado universitario. Manual. A Coruña: Publicaciones de la Revista Galego Portuguesa de Psicoloxia e Educación.

Bigss, J. (1988). Approaches to learning and to essay writing. En R. Schmeck, (Ed.), Learning strategies and learning styles. New York: Plenum Press.

Bigss, J. (1993). What do inventories of students learning processes really measures? A theoretical review and clarification. Brithish Journal of Educational Psychology, 63, 3-19.

Bigss, J. (2005). Calidad del aprendizaje universitario. Madrid: Narcea.

Biggs, J., Kember, D. y Leung, D. (2001). The revised two-factor Study Process Questionnaire: RSPQ-2F. British Journal of Educational Psychology, 71, 133-149.

Cabanach, R.G. (1997). Concepciones y enfoques de aprendizaje. Revista de Psicodidáctica, (4), 5-40. http://www.ehu.es/ojs/index.php/psicodidactica/article/view/56/56 (consultado 15/02/2013).

Castro, A., Ezquerra, P., Argos, J., Osoro, J.M. y Salvador, L. (2012). Dimensionalidad de los enfoques de aprendizaje de los estudiantes universitarios. Actas del V Congreso Mundial de Estilos de Aprendizaje. Santander: Universidad de Cantabria.

De la Fuente, J. y Martínez, J.M. (2003). Cuestionario del proceso de estudio-revisado. Versión castellana. Revised Study Process Questionnaire (R-SPQ-2F) de Biggs, Kember y Leung, 2001. Universidad de Almería.

De Miguel, M. (2005). Modalidades de enseñanza centradas en el desarrollo de competencias. Oviedo: Universidad de Oviedo.

Ezquerra, P., Osoro, J.M., Salvador, L., Argos, J. y Castro, A. (2012). Enfoques de aprendizaje de los estudiantes y su evolución a lo largo de la carrera en función de las metodologías docentes y el contexto institucional. En C. Leite y M. Zabalza (Coords.), Ensino Superior: Innovaçao e qualidade na docência (pp. 1670-1694). Porto: Centro de Investigaçao e Intervençao Educativas (CIIE). 
Fraile, A. y Cornejo, P. (2012). La evaluación formativa en la enseñanza universitaria: una experiencia de innovación educativa con estudiantes de Educación Física. Revista de Evaluación Educativa 1(2). http://revalue.mx/revista/index.php/revalue/issue/current (consultado 15-02-2013).

Gargallo, B. et al. (2009). El cuestionario CEVEAPEU. Un instrumento para la evaluación de las estrategias de aprendizaje de los estudiantes universitarios. RELIEVE 15(2), 1-31. http://www.uv.es/RELIEVE/v15n2/RELIEVEv15n2_5.htm (consultado 15-02-2013).

Gargallo, B. et al. (2011). Actitudes ante el aprendizaje en estudiantes universitarios excelentes y en estudiantes medios. TESI 12(3), 200-229.

Gargallo, B. et al. (2012). Enfoques de Aprendizaje en estudiantes universitarios excelentes y en estudiantes medios. Revista Española de Pedagogía LXX(252), 185-200.

Goicoetxea, J. et al. (2010). Estudio de los contextos de enseñanza y aprendizaje universitarios percibidos por estudiantes y profesores. Propuesta metodológica. I Congreso Internacional Virtual de Formación del Profesorado. La formación del profesorado en el siglo XXI: propuestas antes los cambios económicos, sociales y culturales. http://congresos.um.es/cifop/cifop2010/paper/view/9491/11011 (consultado 15-022013).

Hernández, F. (1999). Los enfoques de aprendizaje en el contexto de la evaluación de la calidad de las universidades (Informe final. Programa sectorial de promoción general del conocimiento. Convocatoria 1995. Ministerio de Educación y Ciencia). Murcia: Universidad de Murcia.

Hernández, F. (2003). Consistencia entre motivos y estrategias de aprendizaje en estudiantes universitarios. Revista de Investigación Educativa, 20(2), 487-510.

Navaridas, F. (2004). Estrategias Didácticas en el aula universitaria. Logroño: Universidad de La Rioja.

Osoro, J.M., Argos, J., Salvador, L., Ezquerra, P. y Castro, A. (2011). La implantación de las titulaciones de Grado de los estudios de Educación: algunas reflexiones y constataciones desde las miradas de docentes y estudiantes. REIFOP, 14(4), 23-30. http://www.aufop.com/aufop/uploaded_files/articulos/1327436135.pdf (consultado 1502-2013).

Salvador, L., Castro, A., Ezquerra, M.P., Osoro, J.M. y Argos, J. (2011). Perfiles de estilos de aprendizaje de los estudiantes universitarios y metodologías docentes. Bordón. Revista de Pedagogía. 63(2), 41-52. Monográfico coord. Por Buela-Casal, G y Teva, I. sobre Enseñanza, aprendizaje y evaluación de la investigación en el Espacio Europeo de Educación Superior.

San Fabián, J.L. et al. (2010). Los contextos de enseñanza y aprendizaje universitarios percibidos por estudiantes y profesores: justificación y marco teórico para su estudio. I Congreso Internacional Virtual de Formación del Profesorado. La formación del profesorado en el siglo XXI: propuestas antes los cambios económicos, sociales y culturales. http://congresos.um.es/cifop/cifop2010/paper/view/11451/10671 (consultado 15-022013).

Recibido: 15 de febrero de 2013

Recepción Modificaciones: 8 de marzo de 2013

Aceptado: 20 de agosto de 2013 